\section{Diagnóstico da Síndrome de Turner: a experiência do Instituto Estadual de Diabetes e Endocrinologia - Rio de Janeiro, de 1970 a 2008}

\author{
Diagnosis of Turner's Syndrome: the \\ experience of the Rio de Janeiro State \\ Institute of Diabetes and Endocrinology \\ between 1970 and 2008
}

Monica de Paula Jung 1 Jorge Luiz do Amaral 2 Rosita Gomes Fontes 3 Aline Teixeira da Costa 4 Susana Maciel Wuillaume 5 Maria Helena Cabral de Almeida Cardoso 6

1,3 Instituto Estadual de Diabetes e Endocrinologia Luiz Capriglione. Rua Moncorvo Filho, 90. Centro. Rio de Janeiro, RJ, Brasil. CEP: 20 211-340. E-mail: monicajung@globo.com

2 Hospital Geral da Santa Casa de Misericórdia do Rio de Janeiro, Rua Santa Luiza, 206, Centro. Rio de Janeiro, RJ. CEP 20020-022 4-6 Instituto Fernandes Figueira/Fundação Oswaldo Cruz. Rio de Janeiro, RJ, Brasil.

\section{Resumo}

Objetivos: descrever a experiência no diagnóstico da Sindrome de Turner (ST), focalizando a distribuição dos cromossomos, a idade, os sinais e sintomas característicos, conforme as fases da vida (lactância, infância, adolescência e adulta).

Métodos: estudo descritivo com 178 pacientes, atendidos de 1970 até 2008. Para análise estatística das diferenças percentuais usou-se o Epi-Info-2000 e para as diferenças entre as médias de idades o teste $t$ de Student e o ANOVA.

Resultados: os cariótipos encontrados foram: 79 com 45,X (35,4\%), 36 com isocromossomo Xq (20,2\%) e 63 com outros mosaicos $(35,4 \%)$. A média de idade do diagnóstico foi de 12,6 anos, sendo menor naquelas com 45,X. Tiveram o diagnóstico feito na lactância 11,3\% das pacientes, 25,3\% na infância, 51,1\% na adolescência e 12,4\% na fase adulta. Daquelas diagnosticadas antes dos cinco anos de idade, 70,6\% apresentaram 45,X. Os sinais que levaram à suspeita diagnóstica na lactância foram o pescoço alado e o linfedema congênito de pés/mãos associados às dismorfias típicas; na infância $e$ adolescência foi a baixa estatura. Cubitus valgus foi encontrado em $72,5 \%$ das pacientes e orelhas anômalas em $65 \%$ das pacientes diagnosticadas com menos de um ano de idade.

Conclusão: o diagnóstico da ST é desnecessariamente atrasado, levando-se em consideração que algumas características típicas podem já estar presentes desde o nascimento.

Palavras-chave Síndrome de Turner, Diagnóstico, Caracteres sexuais, Cariótipo
Key words Turner syndrome, Diagnosis, Sex characteristics, Karyotype 


\section{Introdução}

A Síndrome de Turner (ST) caracteriza-se pela monossomia completa ou parcial do cromossomo X e possui uma grande variabilidade fenotípica, podendo se manifestar na forma clássica - como nos casos descritos por Turner, 1 que relatou sete mulheres, todas elas com retardo do desenvolvimento puberal e do crescimento associados a cubitus valgus, até em casos com poucos sinais dismórficos que possam chamar a atenção ao diagnóstico.

As manifestações clínicas, por vezes sutis, incluem baixa estatura, tórax alargado, hipertelorismo mamário, cubitus valgus, linfedema congênito e falha de desenvolvimento puberal, em função da insuficiência ovariana, sendo que, em alguns casos, malformações de órgãos como o rim e o coração também podem estar presentes. ${ }^{2}$ A ST ocorre aproximadamente em 1:2000 das nascidos vivos. ${ }^{3}$ Essas mulheres têm risco aumentado para diversas co-morbidades, tais como: hipotiroidismo, hipertensão arterial, osteoporose, sobrepeso, déficit auditivo, distúrbios visuais, diminuição da tolerância à glicose e dificuldade de aprendizado. 4

É importante salientar que o retardo do crescimento e a baixa estatura são as duas características mais marcantes. 2 No final da década de 90 , introduziu-se no Brasil o uso do hormônio do crescimento $(\mathrm{GH})$, para tratamento específico da baixa estatura, com a indicação para pacientes com ST, seguindo proposições publicadas em 1992.5 Tal como aponta recente estudo de Davenport et al.,6 que sublinham a importância do diagnóstico precoce da ST, o tratamento com hormônio do crescimento auxilia no aumento da altura e quanto mais cedo for iniciada sua administração melhor será a resposta obtida.

Este estudo tem como objetivo descrever uma experiência de 38 anos no diagnóstico da Síndrome de Turner, no Ambulatório de Genética do Instituto Estadual de Diabetes e Endocrinologia Luiz Capriglione (IEDE). $\mathrm{O}$ estudo focalizou a distribuição dos genótipos cromossômicos, a idade ao diagnóstico e os sinais e sintomas clínicos característicos que foram considerados para realizá-lo.

\section{Métodos}

Estudo descritivo, com dados coletados através de prontuários de crianças, adolescentes e mulheres com Síndrome de Turner, no Ambulatório de Genética do Instituto Estadual de Diabetes e Endocrinologia Luiz Capriglione (IEDE), Rio de Janeiro, RJ, Brasil. Foram incluídos todos os que continham confirmação diagnóstica através de exame citogenético, e cuja data de matrícula no ambulatório foi efetuada de junho de 1970 a setembro de 2008.

De um total de cerca de 67.000 prontuários inscritos no IEDE desde sua fundação em 1969, 205 $(0,31 \%)$ foram registrados como síndrome de Turner. $\mathrm{O}$ atendimento foi feito no Ambulatório de Genética, que conta atualmente com uma equipe multidisciplinar, composta por geneticista clínico, endocrinologista, pediatra, médicos residentes e alunos de pósgraduação em endocrinologia.

Os prontuários selecionados para a pesquisa foram localizados através do arquivo nosológico do Ambulatório de Genética. Dos 205 prontuários, 178 $(86,8 \%)$, estavam disponíveis para consulta. Todos os prontuários continham o exame do cariótipo, sendo que 29 (16,3\%) deles haviam sido feitos fora do Instituto. Foram selecionadas as seguintes variáveis: data do diagnóstico, idade ao diagnóstico, cariótipo, principais características clínicas que motivaram o diagnóstico. Foi construído um banco de dados utilizando-se o software Microsoft Access - versão 2003. As pacientes foram estratificadas em três grupos de acordo com os cariótipos registrados nos prontuários. $\mathrm{O}$ primeiro composto pelas pacientes com carótipo 45,X; o segundo por cariótipos com isocromossomo do braço longo do $\mathrm{X}$ (iXq) e o último incluiu os demais cariótipos [mosaicos com material do cromossomo $\mathrm{Y}$ e mosaicos como 45,X/46,XX, 45X/46,X,r(X)]. As pacientes foram distribuídas de acordo com a fase da vida: lactância (até um ano de vida), infância (maior de um ano até 11 anos), adolescência (de 12 a 18 anos) e fase adulta (maior de 18 anos).

$\mathrm{Na}$ análise estatística, para estabelecer se as diferenças nos percentuais entre os três subgrupos eram estatisticamente significativas, foi usado o teste de comparação de proporções do Epitable, do programa Epi-info 2000. Para estabelecer a diferença entre as médias de idade foram usados o teste $\mathrm{t}$ Student e o teste ANOVA, com pós-teste de Bonferroni do programa GraphPad Prism versão 4.00 para Windows, GraphPad Software. Em todos os casos foi considerado como estatisticamente significativo o $p<0,05$.

Este estudo seguiu o protocolo da resolução 196/1996 do Conselho Nacional de Saúde, tendo sido aprovada pelo Comitê de Ética do Instituto Estadual de Endocrinologia e Diabetes Luiz Capriglione, sob parecer no 306 de 19 de dezembro de 2006. 


\section{Resultados}

Do total dos 178 casos estudados, 79 tinham o cariótipo 45,X; 36 apresentavam cariótipo incluindo um isocromossomo Xq [iX(q)], mosaico ou não, e os 63 restantes mosaicos e outros (Tabela 1).

Dentro do grupo dos $63(35,4 \%)$ cariótipos mosaicos, observamos 14 (7,9\%) com cariótipo $45, \mathrm{X} / 46, \mathrm{X} \quad \mathrm{r}(\mathrm{X})$ e $10 \quad(5,6 \%)$ com cariótipo 45,X/46,XX.

$\mathrm{Na}$ Figura 1 são descritos os genótipos cromossômicos associados a ST. Quando distribuídos de acordo com a faixa etária à época do diagnóstico, mostram que abaixo dos cinco anos predomina o cariótipo $45, \mathrm{X}$, correspondente a 70,6\% (24/34); ao passo que, considerando o total das 178 pacientes estudadas, esse cariótipo apresenta-se em $44,4 \%$.
A média de idade quando do diagnóstico foi de $12,59 \pm 0,61$ anos (IC95\%: 11,39-13,78), sendo que as idades variaram de 1 dia a 48 anos.

Das 178 pacientes, $20(11,2 \%)$ tiveram seu diagnóstico na lactância, $45(25,3 \%)$ na infância, 91 $(51,1 \%)$ na adolescência e $22(12,4 \%)$ na fase adulta.

Ao se avaliar a associação do cariótipo com a idade ao diagnóstico, para as pacientes com cariótipo clássico 45,X, a média de idade foi de 10,62 $\pm 1,04$ anos (IC95\%: 8,55-12,68); para as com cariótipo contendo um iX(q) foi de 15,25 $\pm 0,77$ anos (IC95\%: 13,68-16,82); e no grupo de mosaicos/outros a média de idade ao diagnóstico foi de 13,53 $\pm 0,96$ anos (IC95\%: 11,61-15,45). Essas diferenças foram estatisticamente significativas, sendo que a média de idade ao diagnóstico do grupo $45, \mathrm{X}$ foi diferente da média de idade do grupo iX(q) $(p<0,05)$ Figura 2.

Tabela 1

Distribuição dos cariótipos: monossomia de $\mathrm{X}$, isocromossomo de $\mathrm{Xq}$ e mosaicos e outros $(\mathrm{N}=178)$.

\begin{tabular}{lll}
\hline Cariótipos & $\mathbf{N}$ & $\%$ \\
\hline $45, X$ & 79 & 44,4 \\
& & 20,2 \\
$45, X / 46, i(X q) ; 46, X i(X q)$ & 36 & 35,4 \\
$45, X / 47, i(X q), i(X q), i(X q)$ & 63 & \\
$45, X / 46, X X ; 45, X / 46, X X / 47, X X X ; 45, X / 47, X X X$ & \\
$45, X / 46, X, r(X) ; 45, X / 46, X, d e l(X p) ; 46, X, \operatorname{del}(X q)$ & \\
$45, X / 46, X, i(X p) ; 45, X / 46, X+\operatorname{mar}$ & & \\
$45, X / 46, X Y ; 45, X / 46, X, d e l(Y) ; 45, X / 46, X, r(Y)$ & & \\
e outros & & \\
\hline
\end{tabular}

Figura 1

Distribuição dos cariótipos de acordo com a idade ao diagnóstico ( $\mathrm{N}=178)$.

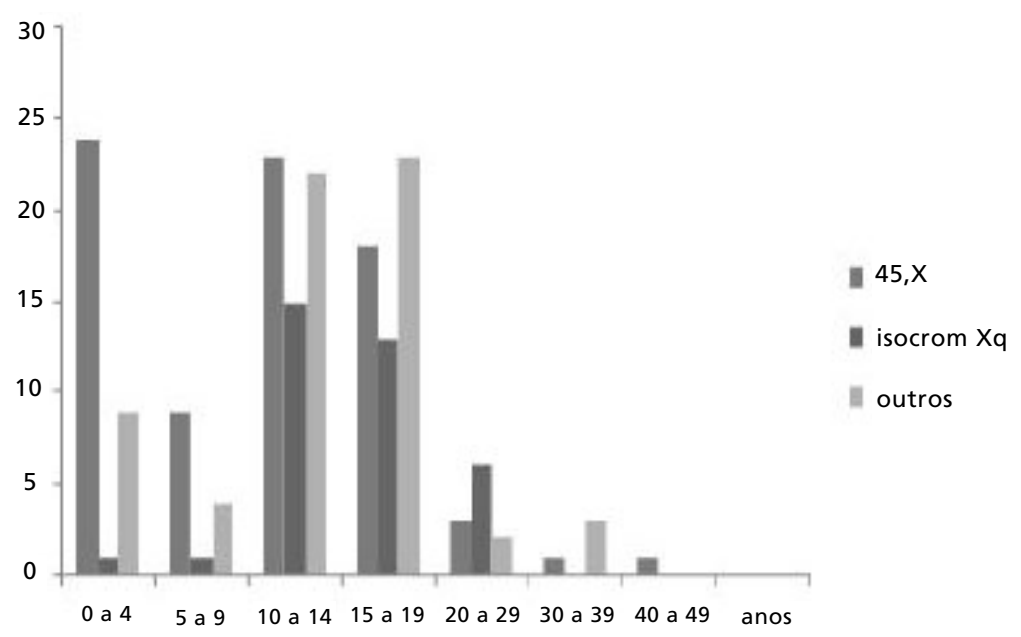




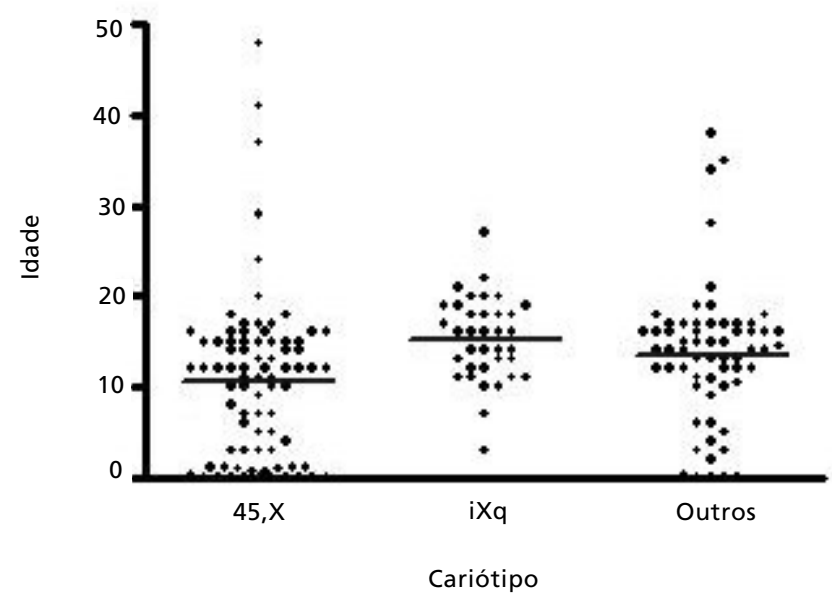

Nota: cada ponto representa a idade de uma mulher ao diagnóstico. As linhas horizontais representam a média de idade do grupo conforme o cariótipo.

O cariótipo $45, \mathrm{X}$ foi encontrado em $75 \%(15 / 20)$ das meninas cujo diagnóstico foi feito durante a lactância, em 53,3\% (24/45) daquelas diagnosticadas na infância, em 37,4\% (34/91) daquelas diagnosticadas na adolescência e em $22,7 \%(5 / 22)$ das mulheres diagnosticadas na idade adulta.

Os sinais e sintomas apresentados variaram conforme a idade de diagnóstico. Nas pacientes diagnosticadas na idade de lactente o pescoço alado estava presente em 13/20 e o linfedema congênito de pés ou mãos, associados com dismorfias típicas em $15 / 20$, sendo essas as principais razões para o diagnóstico (Tabela 2).

Tabela 2

Dismorfias e características clínicas típicas da Síndrome de Turner, de acordo com a idade da confirmação do diagnóstico. (N=178)

\begin{tabular}{|c|c|c|c|c|c|c|c|c|c|c|}
\hline \multirow[t]{2}{*}{ Características clínicas } & \multicolumn{2}{|c|}{$\begin{array}{l}\text { Lactância } \\
(\mathrm{N}=\mathbf{2 0})\end{array}$} & \multicolumn{2}{|c|}{$\begin{array}{l}\text { Infância } \\
(\mathrm{N}=45)\end{array}$} & \multicolumn{2}{|c|}{$\begin{array}{l}\text { Adolescência } \\
\qquad(\mathrm{N}=91)\end{array}$} & \multicolumn{2}{|c|}{$\begin{array}{l}\text { Adulta } \\
(\mathrm{N}=20)\end{array}$} & \multicolumn{2}{|c|}{$\begin{array}{c}\text { Total } \\
(\mathrm{N}=178)\end{array}$} \\
\hline & $\mathrm{n}$ & $\%$ & $\mathrm{n}$ & $\%$ & $n$ & $\%$ & $\mathrm{n}$ & $\%$ & $\mathrm{n}$ & $\%$ \\
\hline Otite de repetição & 3 & 15,0 & 8 & 17,8 & 14 & 15,4 & 2 & 9,1 & 27 & 15,2 \\
\hline Palato ogival & 13 & 65,0 & 25 & 55,6 & 55 & 60,4 & 13 & 59,1 & 106 & 59,6 \\
\hline Atraso puberal & 0 & - & 0 & - & 41 & 45,1 & 17 & 77,3 & 58 & 52,3 \\
\hline Unhas displásicas & 3 & 15,0 & 3 & 6,7 & 13 & 14,3 & 0 & - & 19 & 10,7 \\
\hline Orelhas anômalas & 13 & 65,0 & 16 & 35,6 & 28 & 30,8 & 4 & 18,2 & 61 & 34,3 \\
\hline Retrognatia & 3 & 15,0 & 3 & 6,7 & 13 & 14,3 & 4 & 18,2 & 23 & 12,9 \\
\hline Cubitus valgus & 8 & 40,0 & 27 & 60,0 & 67 & 73,6 & 17 & 77,3 & 129 & 72,5 \\
\hline Hipertelorismo mamário & 8 & 40,0 & 18 & 40,0 & 46 & 50,6 & 8 & 36,4 & 81 & 45,5 \\
\hline Tórax alargado & 6 & 30,0 & 9 & 20,0 & 29 & 31,9 & 6 & 27,3 & 50 & 28,1 \\
\hline Epicanto & 7 & 35,0 & 9 & 20,0 & 14 & 15,4 & 5 & 22,7 & 35 & 19,7 \\
\hline Baixa implantação de cabelos & 10 & 50,0 & 18 & 40,0 & 41 & 45,1 & 9 & 40,9 & 78 & 43,8 \\
\hline Pescoço alado ou alargado & 13 & 65,0 & 18 & 40,0 & 27 & 29,7 & 6 & 27,3 & 64 & 36,0 \\
\hline
\end{tabular}


Dismorfias e características clínicas típicas da Síndrome de Turner, de acordo com a idade da confirmação do diagnóstico. (N=178)

\begin{tabular}{|c|c|c|c|c|c|c|c|c|c|c|}
\hline \multirow[t]{2}{*}{ Características clínicas } & \multicolumn{2}{|c|}{$\begin{array}{l}\text { Lactância } \\
(\mathrm{N}=20)\end{array}$} & \multicolumn{2}{|c|}{$\begin{array}{l}\text { Infância } \\
(\mathrm{N}=45)\end{array}$} & \multicolumn{2}{|c|}{$\begin{array}{l}\text { Adolescência } \\
\qquad(\mathrm{N}=91)\end{array}$} & \multicolumn{2}{|c|}{$\begin{array}{l}\text { Adulta } \\
(\mathrm{N}=20)\end{array}$} & \multicolumn{2}{|c|}{$\begin{array}{c}\text { Total } \\
(\mathrm{N}=178)\end{array}$} \\
\hline & $\mathrm{n}$ & $\%$ & $\mathrm{n}$ & $\%$ & $\mathrm{n}$ & $\%$ & $\mathrm{n}$ & $\%$ & $\mathrm{n}$ & $\%$ \\
\hline Escoliose & 2 & 10,0 & 3 & 6,7 & 11 & 12,1 & 6 & 27,3 & 22 & 12,4 \\
\hline 40 metacarpiano curto & 6 & 30,0 & 17 & 37,8 & 47 & 51,7 & 7 & 31,8 & 78 & 43,8 \\
\hline 40 metatarsiano curto & 3 & 15,0 & 2 & 4,4 & 11 & 12,1 & 11 & 50,0 & 19 & 10,7 \\
\hline Ptose palpebral & 1 & 5,0 & 2 & 4,4 & 13 & 14,3 & 1 & 4,6 & 17 & 9,6 \\
\hline Linfedema pés /mãos & 8 & 40,0 & 7 & 15,6 & 3 & 3,3 & 0 & - & 18 & 10,1 \\
\hline Malformação cardíaca & 7 & 35,0 & 1 & 2,2 & 4 & 4,4 & 0 & - & 12 & 6,7 \\
\hline Pectus escavatum & 1 & 5,0 & 4 & 8,9 & 7 & 7,7 & 0 & - & 12 & 6,7 \\
\hline Nevus & 5 & 25,0 & 15 & 33,3 & 40 & 43,9 & 6 & 27,3 & 58 & 32,6 \\
\hline Hipertelorismo ocular & 2 & 10,0 & 3 & 6,7 & 10 & 11,0 & 2 & 9,1 & 17 & 9,6 \\
\hline
\end{tabular}

\section{Discussão}

Nossa casuística mostra uma frequência de cariótipos similar ao descrito na literatura especializada. Hook e Warburton, 7 numa série de 902 pacientes com ST, encontraram 53\% com cariótipo $45, \mathrm{X}, 16,8 \%$ com um $\mathrm{i}(\mathrm{Xq})$ e $33 \%$ com mosaicos ou outros tipos de cariótipos. Uma coorte de 3425 pacientes com ST, relatou frequência de cariótipo $45, \mathrm{X}$ de $36 \%, 18,3 \%$ com um $\mathrm{i}(\mathrm{Xq})$, e $46 \%$ para outros cariótipos mosaicos. 8 El-Mansoury et al. 9 também mostraram que a frequência maior de cariótipos é de 45,X (48\%), sendo que 14\% possuíam cariótipo com um $\mathrm{i}(\mathrm{Xq})$ e $38 \%$ outros cariótipos ou mosaicos.

Segundo a literatura especializada, 99\% das gestações com fetos com ST abortam espontaneamente, o que indica que as pacientes com cariótipo 45 , X podem ser mosaicos em alguma linhagem ou o foram em alguma fase da vida. 10 Nas nossas pacientes todos os cariótipos foram feitos em linfócitos de sangue periférico, mas pode existir mosaicismo em outros tecidos diferentes dos linfócitos, e que se fossem realizadas análises, principalmente em fibroblastos e gônadas, provavelmente encontraríamos maior número de pacientes com mosaicismos. ${ }^{11}$ Isso é o que mostra um estudo12 com dez meninas com baixa estatura idiopática, que pesquisou mosaicismos crípticos, através da utilização de técnicas citogenéticas como bandeamento (GTG) e fluorescent in situ hibridization (FISH) com sondas alfacentroméricas para X ou Y em células de mucosa oral, e encontrou um caso de mosaicismo 45,X/46, X, der(X)/46,X,r(X) e outros dois com cariótipo normal, no sangue periférico, porém com mosaicismo críptico $45, \mathrm{X} / 46, \mathrm{XX}$ ao exame das células de mucosa oral. Em 2008,11 a utilização de técnicas de biologia molecular com o ensaio do human androgen receptor chromosome inactivation assay (HUMARA) pôde revelar a presença de um segundo cromossomo X em $25 \%$ de 36 casos de pacientes com cariótipo 45,X.

Todos os exames de cariótipos do nosso estudo foram feitos pós-nascimento. Não houve registro de diagnóstico intraútero, via ultrassonografia, apenas o de uma suspeita confirmada no pós-natal imediato. A literatura sobre ST, desde meados da década de 1995, destaca que o diagnóstico pré-natal é geralmente um achado casual, via imagem ultrassonográfica, que pode mostrar, por exemplo, higroma cístico e cardiopatia congênita. ${ }^{13}$

As anomalias esqueléticas, tais como cubitus valgus e quarto metacarpiano curto, encontradas nas mulheres com ST, podem ser atribuídas à haploinsuficiência do gene short stature homeobox-containing (SHOX).14,15 O cubitus valgus, encontrado em $72,5 \%$ dos casos, foi uma das características mais frequentes nas nossas 178 pacientes. No grupo de pacientes acima de 13 anos de idade ao diagnóstico, encontramos um percentual ainda mais elevado, de $75,7 \%$, ao passo que, quando nos detivemos no grupo diagnosticado quando lactente, o percentual foi de $40 \%$. Tal fato chama a atenção e faz pensar sobre a importância de, durante o primeiro ano de vida, avaliar o ângulo do cotovelo como possível pista e/ou indício de ST. Não se pode considerar o cubitus valgus isoladamente como um sinal patognomônico, porém o trabalho de Sävendahl e 
Davenport 16 relata a presença significante de cubitus valgus em $52 \%$ de 25 pacientes diagnosticadas na lactância. Em concordância com trabalho de Rappold et al., ${ }^{17}$ esse é, sem dúvida, um sinal que aponta para a presença de uma provável haploinsuficiência do gene SHOX como o que ocorre na ST.

A presença do palato ogival em $50 \%$ das pacientes diagnosticadas na fase adulta também é um importante sinal, assim como a presença dos metatarsos curtos, que na nossa casuística ocorreu em $50 \%$ dos casos. No que diz respeito à presença do quarto metacarpiano curto, o guideline proposto por Sävendahl e Davenport 16 o incorpora como um dos sinais a ser observados para o diagnóstico precoce. As orelhas anômalas foram mais observadas nas menores idades, sendo ainda muito mais frequentes nas pacientes diagnosticadas com menos de um ano de idade (65\%).

O diagnóstico da ST é, em geral, desnecessariamente atrasado. Somente $11 \%$ das pacientes foram diagnosticadas na fase da lactância. O restante (158) teve o diagnóstico com idade média de 13,9 anos, apesar de a maioria apresentar déficit do crescimento e características típicas da ST. As pacientes identificadas no período da lactância foram diagnosticadas por apresentarem, quase todas, linfedema congênito de mãos e pés, associados com outras dismorfias. Além disto, na maior parte delas, também se registrou a presença de características secundárias ao linfedema, como pescoço alado, baixa implantação dos cabelos na nuca, orelhas anômalas ou baixo implantadas e unhas displásicas. Ressaltamos, contudo, que apesar de apresentarem essas características clássicas, as mesmas não foram valorizadas à época do nascimento. Das pacientes diagnosticadas na infância, 15,5\% reportaram história de linfedema congênito, tendo, portanto, sido perdida a chance de se fazer um diagnóstico mais precoce. A mesma omissão ocorreu em relação ao pescoço alado, que apesar de ter sido observado desde o nascimento em $29 \%$ dos casos, esses só foram diagnosticados na fase da adolescência. Por outro lado, somando-se as 136 pacientes com diagnóstico na infância e na adolescência, dez (7,35\%) informaram ter tido linfedema ao nascer. Nossos resultados mostraram uma média de idade de diagnóstico de 12,6 anos, que poderia ser considerada como tardia, tendo em vista que os sinais principais estavam presentes desde o nascimento. Neste estudo $72,8 \%$ das pacientes que tiveram o diagnóstico acima dos 18 anos de idade relataram história de atraso puberal, tornando esse sintoma um dos mais frequentes para o diagnóstico quando da entrada na vida adulta. Contudo, estudos como o realizado na Grã Bretanha, que relata uma média de 14,5 anos de idade ao diagnóstico. ${ }^{8}$ A base de dados do Belgian Study Group for Pediatric Endocrinology, 18 mostra que a média de idade ao diagnóstico, em 1991, era de 11,2 anos e que, em 2003, era 6,6 anos. Apesar disso, pode-se afirmar que o retardo no diagnóstico continua sendo um problema a ser resolvido, apontando para a necessidade de neonatologistas e pediatras mostrarem-se atentos às possibilidades da existência de síndromes genéticas como a Síndrome de Turner.

A média de idade de diagnóstico nas pacientes com cariótipo $45, \mathrm{X}$ foi significativamente menor do que naquelas com cariótipos $\mathrm{i}(\mathrm{Xq})(p<0,05)$. Esses achados são semelhantes aos de estudos realizados na Dinamarca, 2 que registraram, respectivamente, média de idade ao diagnóstico de 13, 14 e 19 anos para as mulheres com cariótipo 45,X, i(Xq) e outros cariótipos. Não é surpresa que pacientes com cariótipos 45, $\mathrm{X}$ tenham tido um diagnóstico mais precoce, pois elas, em geral, exibem mais estigmas. Entretanto, estudos têm mostrado que mulheres com $\mathrm{ST}$, com cariótipos mosaicos ou outros mais raros, têm a altura tão reduzida quanto as com cariótipo 45,X. A maioria delas também exibe falência ovariana prematura e a demora no diagnóstico é bastante preocupante, sendo necessário que se aumente a vigilância da síndrome para as meninas com baixa estatura. 19

A baixa estatura esteve presente em quase $97 \%$ dos casos, tendo sido o fator que desencadeou o diagnóstico na maioria deles, tanto no período da infância quanto da adolescência. Infelizmente, essas pacientes tinham ao tempo de diagnóstico uma estatura em torno de mais dois desvios padrões (DP) abaixo da média para a idade. Achados semelhantes foram relatados em diversos estudos: em grupo de pacientes com ST cujo diagnóstico foi realizado na média idade de 12 anos e cuja estatura era de -3 DP se comparada a meninas da mesma idade; 19 em uma série de pacientes referidas para avaliação genética por baixa estatura isolada, na qual se registrou que $4 \%$ delas tinham $\mathrm{ST}^{20}$ e, em análise realizada por Sävendahl e Davenport, 16 com 85 pacientes com ST, que mostrou uma demora no diagnóstico de em média 7,7 anos para as diagnosticadas na adolescência, com uma média discretamente menor, de 5,3 anos, para aquelas que haviam caído abaixo do 50 percentil de altura. Se consideradas as características étnicas da população sul-americana, o retardo do crescimento e a baixa estatura, quando não tratados, podem levar à estatura adulta variando em torno de $144,8 \mathrm{~cm}$, segundo um estudo em pacientes brasileiras 21 ou até $138,2 \mathrm{~cm}$, como encontrado num estudo realizado no Chile. 22 
O retardo no diagnóstico implicará no atraso do início do tratamento. O uso adequado do $\mathrm{GH}$ pode aumentar de $3,9 \mathrm{~cm}$ a $8,5 \mathrm{~cm}$ a altura prevista. 23,24 No Japão, a avaliação de 1478 pacientes com ST, que fizeram uso de $\mathrm{GH}$, mostrou que a média de idade e o DP da altura ao iniciar o tratamento naquelas com menos de cinco anos de idade aumentou significativamente com o passar do tempo. ${ }^{25}$ Entretanto, essa tendência favorável ainda não tem preenchido, completamente, as condições recomendadas pelos guidelines internacionais para ST.26 Segundo dados do Pharmacia and Upjohn International Database, ${ }^{27}$ no Reino Unido, a média de idade para o início do tratamento com $\mathrm{GH}$ foi reduzida de 10,4 em 1986 para 8,5 anos em 1996. Está claro que o crescimento é subnormal desde o nascimento, 28,29 e por isso foi realizado um estudo randomizado, controlado e multicêntrico para o uso de $\mathrm{GH}$ em crianças com ST, a partir de nove meses de idade, cujo resultado evidenciou que o quanto antes se iniciar o tratamento menor será a perda na estatura. 6

\section{Referências}

1. Turner HH. A syndrome of infantilism, congenital webbed neck, and cubitus valgus. Endocrinol. 1938; 23: 566-74.

2. Jones KL, Smith DW. Turner Syndrome. In: Smith's recognizable patterns of human malformation. Philadelphia: Elsevier Sauders; 2006. p. 76-81.

3. Stochholm K, Juul S, Juel K, Naeraa RW, Gravholt $\mathrm{CH}$. Prevalence, incidence, diagnostic delay, and mortality in Turner syndrome. J Clin Endocrinol Metab. 2006; 91: 3897-902.

4. Gravholt CH, Juul S, Naeraa RW, Hansen J. Morbity in Turner syndrome. J Clin Epidemiol. 1998; 51: 147-58.

5. Rosenfeld RG, Frane J, Attie KM, Brasel JA, Burstein S, Cara JF, Chernausek S, Gotlin RW, Kuntze J, Lippe BM, Mahoney PC, Moore VW, Saenger P, Johanson AJ. Six-year results of a randomized, prospective trial of human growth hormone and oxandrolone in Turner syndrome. J Pediatr (Rio J). 1992; 121: 49-55

6. Davenport ML, Crowe BJ, Travers SH, Rubin K, Ross JL, Fechner PY, Gunter DF, Liu C, Geffner M, Thrailkill K, Huseman C, Zagar AJ, Quiqley C. Growth hormone treatment of early growth failure in toddlers with Turner Syndrome: a randomized, controlled, multicenter trial. J Clin Endocrinol Metab. 2007; 92: 3406-16.

7. Hook EB, Warburton D. The distribution of chromosomal genotypes associated with Turner's Syndrome: livebirth prevalence rates and evidence for diminished fetal mortality and severity in genotypes associated with structural $\mathrm{X}$ abnormalities or mosaicism. Hum Genet. 1983; 64: 24-27.

8. Schoemaker MJ, Swerdlow AJ, Higgins CD, Wright AF, Jacobs PA. Cancer incidence in women with Turner syndrome in Great Britain: a national cohort study. Lancet Oncol. 2008; 9: 239-46.
O mais recente guideline prático produzido pelo Turner Syndrome Consensus Study Group 26 recomenda que o objetivo dos tratamentos promotores do crescimento seja alcançar a altura normal tão cedo quanto possível. Também postula que o diagnóstico da ST deveria ser considerado em qualquer paciente do sexo feminino, com diminuição de crescimento não explicada, atraso de puberdade ou quaisquer outros estigmas tais como edema das mãos ou pés, pescoço alado ou anomalias cardíacas do coração esquerdo. 26

Os resultados desse estudo mostraram-se compatíveis com os relatados na literatura mundial sobre a ST, reforçando o retardo no diagnóstico.

\section{Agradecimentos}

A Thiago Moraes pela colaboração na elaboração do banco de dados e Martha Mutis pelo auxílio na análise estatística.
9. El-Mansoury M, Barrenäs ML, Bryman I, Hanson C, Larsson C, Wilhelmsen L, Landin-Wilhelmsen K. Chromosomal mosaicism mitigates stigmata and cardiovascular risk factors in Turner syndrome. Clin Endocrinol. 2007; 66: 744-51.

10. Hassold T, Benhaham F, Leppert M. Cytogenetics and molecular analysis of sex chromosome monossomy. Am J Hum Genet. 1988; 42: 534-41.

11. Araújo A, Ramos ES. Cryptic mosaicism involving a second chromosome $\mathrm{X}$ in patients with Turner syndrome. Braz J Med Biol Res. 2008; 41: 368-72.

12. Martins RRS, Ramos HIB, Llerena Jr. JC, Almeida JCC. Investigação clínica e genética em meninas com baixa estatura idiopática. Arq Bras Endocrinol Metab. 2003; 47: 684-94.

13. Berdahl LD, Wenstrom KD, Hanson JW. Web neck anomaly and its association with congenital heart disease. Am J Med Genet. 1995; 10; 56: 304-7.

14. Clement-Jones M, Schiller S, Rao E, Blaschke RJ, Zuniga A, Zeller R, Robson SC, Binder G, Glass I, Strachan T, Lindsey S, Rappold GA. The short stature homeobox gene SHOX is involved in skeletal abnormalities in Turner syndrome. Hum Mol Genet. 2000; 9: 695-702.

15. Kosho T, Muroya K, Nagai T, Fugimoto M, Yokoya S, Sakamoto H, Hirano T, Terasaki H, Ohashi H, Nishimura G, Sato S, Matsuo N, Ogata T. Skeletal features and growth patterns in 14 patients with haploinsufficiency of SHOX: Implications for the development of Turner syndrome. J Clin Endocrinol Metab. 1999; 84: 4613-21.

16. Savendahl L, Davenport ML. Delayed diagnoses of Turner's syndrome: Proposed guidelines for change. J Pediatr (Rio J). 2000; 137: 455-9. 
17. Rappold GA, Blum WF, Shavrikova EP, Crowe BJ, Roeth R, Quigley CA, Ross JL, Niesler B. Genotypes and phenotypes in children with short stature: clinical indicators of SHOX haploinsufficiency. J Med Genet. 2007; 44: 306-13.

18. Massa G, Verlinde F, De Shepper J, Thomas M, Bourgignon JP, Craen M, de Zegher F, Francois I, Du Caju M, Maes M, Heinrichs C, The Belgian Study Group for Paediatric Endocrinology. Trends in age at diagnosis of Turner syndrome. Arch Dis Child. 2005; 90: 267-8.

19. Massa GG, Vanderschueren-Lodeweyckx M. Age and height at diagnosis in Turner syndrome: influence of parental height. Pediatrics. 1991; 88: 1148-52.

20. Moreno-García, M, Martínez, FJF, Miranda EB. Chromosomal anomalies in patients with short stature. Pediatr Interl. 2005; 47: 546-9.

21. Lemos-Marini SH, Morcillo AM, Baptista MT, Guerra-Jr G, Maciel-Guerra AT. Spontaneous final height in Turner's syndrome in Brazil. J Pediatr Endocrinol Metab. 2007; 20: 1207-14.

22. Román R, Vallejos ME, Muñoz M, Schneider R, Youlton R, Henriquez C, Cassorla F. Turner's syndrome: growth and clinical description of 83 Chilean girls. Rev Med Chil. 2002; 130: 977-84.

23. Soriano-Guillén L, Coste J, Ecosse E, Léger J, Tauber M, Cabrol S, Nicolino M, Brauner R, Chaussain JL, Carel JC. Adult height and pubertal growth in Turner syndrome after treatment with recombinant growth hormone $\mathrm{J}$ Clin Endocrinolo Metab. 2005; 90: 5197-204.

Recebido em 11 de fevereiro de 2009

Versão final apresentada em 21 de setembro de 2009

Aprovado em 23 de outubro de 2009
24. Ramos AV, Silva IN, Goulart EMA. Turner syndrome: searching for better outcomes. Clinics. 2008; 63: 173-8.

25. Isojima T, Yokoya S, Ito J, Horikawa R, Tanaka T. Trends in Age and Anthropometric Data at Start of Growth Hormone Treatment for Girls with Turner Syndrome in Japan. [Accessed 26 out 2008]. Available in: http://www.jstage.jst.go.jp/ article/endocrj/ advpub/0/ advpub_0808250186/_article

26. Bondy CA. Care of girls and women with Turner syndrome: a guideline of the Turner syndrome study group. J Clin Endocrinol Metab. 2007; 92: 10-25.

27. Betts PR, Butler GE, Donaldson MDC, Dunger DB, Johnston DI, Kelnar CJH, Krik J, Price DA, Wilton P. A decade of growth hormone treatment in girls with Turner syndrome in the UK. UK KIGS Executive Group. Arch Dis Child. 1999; 80: 221-5.

28. Rongen-Westerlaken C, Corel L, Van den Broeck J, Massa G, Kalberg J, Albertsson-Wikland,K Naeraa RW, Wit JM. Reference values for height, height velocity and weight in Turner's syndrome. Acta Paediatr. 1997; 86: 937-42.

29. Davenport ML, Punyasavatsut N, Stewart PW, Gunther DF, Sävendahl L, Sybert VP. Growth failure in early life: An important manifestation of Turner Syndrome. Horm Res. 2002; 57: 157-64. 